\title{
Medicina de Família e Comunidade: breve histórico, desafios e perspectivas na visão de discentes de graduação
}

\author{
Family and Community Medicine: a brief history, challenges, and perspectives in the view of \\ undergraduate students
}

\section{Medicina Familiar y Comunitaria: breve historia, desafíos y perspectivas desde la visión de los estudiantes de pregrado}

\author{
Vinicius Gomes de Oliveira. Fundação Universidade Federal do Tocantins (UFT). Palmas, TO, Brasil. viniciusgomes@uft.edu.br (Autor correspondente) \\ Fernanda Navarini Queiroz. Fundação Universidade Federal do Tocantins (UFT). Palmas, TO, Brasil. fenavarini@hotmail.com \\ Bruno Pereira Araújo. Fundação Universidade Federal do Tocantins (UFT). Palmas, TO, Brasil. brunimipatinga@hotmail.com \\ Cássio Marques Menezes Silva. Fundação Universidade Federal do Tocantins (UFT). Palmas, TO, Brasil. cassiomarkenze@hotmail.com \\ Flávio Dias Silva. Fundação Universidade Federal do Tocantins (UFT). Palmas, TO, Brasil. flaviodias@uft.edu.br
}

\section{Resumo}

A Medicina de Família e Comunidade (MFC) é fundamentada em princípios que estão diretamente vinculados à Atenção Primária à Saúde (APS), que é a base de um sistema para otimizar recursos e serviços em saúde. 0 presente artigo procura analisar a atual conjuntura da MFC na visão de discentes de graduação em medicina, com enfoque na APS. Faz-se, então, um breve resgate dos principais eventos históricos que nortearam a criação dessa especialidade no Brasil, tais como conferências, programas de Residência Médica e criação do Sistema Único de Saúde (SUS). Por fim, retorna-se aos dias de hoje, para se estabelecer uma conexão entre as propostas iniciais e o contexto atual da saúde, oferecendo uma visão crítica acerca desses aspectos. Procura-se destacar o programa Mais Médicos e o Programa de Valorização da Atenção Básica (PROVAB), ambos propostos recentemente pelo governo federal, com 0 intuito de corroborar as estratégias traçadas há algumas décadas.

\section{Abstract}

Family and Community Medicine (FCM) is grounded on principles that are directly linked to Primary Health Care (PHC), which is the basis of a system to optimize resources and health services. In this article, we analyze the current situation of FCM in the view of undergraduate medical students with a focus on PHC. We then present a brief account of the major historical events that guided the creation of this specialty in Brazil, such as conferences, medical residency programs, and the inception of the Unified Health System (SUS). Finally, we return to our present time to establish a connection between the initial proposals and the current context of health care, offering a critical view of these aspects. We seek to highlight the "Mais Médicos" programme and the "Programa de Valorização da Atenção Básica" (PROVAB), both recently launched by the Brazilian Federal Government, in order to corroborate the strategies outlined a few decades ago.

\section{Resumen}

La Medicina Familiar y Comunitaria (MFC) se fundamenta en principios que están directamente vinculados a la Atención Primaria de Salud (APS), que es la base de un sistema para optimizar los recursos y servicios de salud. En este artículo se analiza la situación actual del MFC desde la visión de los estudiantes de pregrado en medicina, con un enfoque en la APS. A continuación se hace un breve rescate de los principales acontecimientos históricos que guiaron la creación de esta especialidad en Brasil, tales como conferencias, programas de residencia médica y la creación del Sistema Único de Salud (SUS). Por último, se vuelve a la actualidad, para establecer una conexión entre las propuestas iniciales y el contexto actual de la salud, ofreciendo una visión crítica de estos aspectos. Se pretende dar a conocer el programa Más Médicos y el Programa de Valorización de la Atención Básica (PROVAB), ambos propuestos recientemente por el Gobierno Federal Brasileño, con el fin de corroborar las estrategias esbozadas hace algunas décadas.

Como citar: Oliveira VG, Queiroz FN, Araúio BP, Silva CMM, Silva FD. Medicina de Família Comunidade: breve histórico, desafios e perspectivas na visão de discentes de graduação. Rev Bras Med Fam Comunidade. 2014;9(30):85-8.Disponível em: http://dx.doi.org/10.5712/rbmfc9(30)850

\section{Palavras-chave:}

Medicina de Família e

Comunidade

Atenção Primária à Saúde

Sistema Único de Saúde

Políticas, Planejamento e

Administração em Saúde

\section{Keywords:}

Family Practice

Primary Health Care Unified Health System Health Policy, Planning and Management

\section{Palabras clave:} Medicina Familiar y Comunitaria Atención Primaria de Salud Sistema Único de Salud Políticas, Planificación y Administración en Salud

\section{Fonte de financiamento:} declaram não haver.

Parecer CEP:

não se aplica.

Conflito de interesses:

declaram não haver. 


\section{Introdução}

A Atenção Primária à Saúde (APS) é a base organizacional de um sistema que busca otimizar recursos e serviços em saúde. Tem como ponto central a prevenção de doenças, bem como a promoção e a manutenção da saúde por meio de uma prestação de serviços continuada, centrada no indivíduo e no contexto em que ele está inserido. Funciona como o primeiro contato da população com o sistema de saúde, por meio do qual esta será direcionada aos demais níveis de atenção, caso necessário. ${ }^{1}$

A Medicina de Família e Comunidade (MFC) é uma especialidade que se fundamenta em princípios que envolvem a atuação na APS, a atenção centrada na pessoa, a educação médica continuada, o humanismo e a formação de liderança. Trata-se, portanto, de uma área médica fundamental para o bom desenvolvimento da APS e consequentemente para a consolidação de um sistema de saúde resolutivo, abrangente e capaz de gerenciar de forma eficaz seus recursos. ${ }^{2,3}$

Este artigo tem como objetivo apresentar um breve histórico e o contexto atual da MFC no Brasil, bem como discutir pontos-chave para a qualificação da APS, a fim de oferecer um atendimento resolutivo à população.

\section{Breve histórico da Medicina de Família e Comunidade no Brasil}

A MFC teve início no Brasil em meio a um modelo médico voltado para o atendimento hospitalar e um crescimento das subespecializaçôes. Inicialmente marginalizada, a MFC ganhou maior visibilidade após a expansão do Programa Saúde da Família. ${ }^{4}$ A MFC não é uma novidade no Brasil ou no mundo e, em busca de se entender o seu processo de formaçáo, faz-se necessário um breve resgate de sua história.

A necessidade de aperfeiçoamento para suprir as carências da população nos níveis primários da atenção à saúde fez com que, em 1976, fosse criado o Programa de Residência Médica (PRM) em Medicina Geral e Comunitária, atual Medicina de Família e Comunidade. Os projetos pioneiros foram o do Centro de Saúde Escola Murialdo, em Porto Alegre-RS; o do Projeto Vitória de Santo Antão, em Santo Antão-PE; e o do Serviço de Medicina Integral da Universidade do Estado do Rio de Janeiro (UERJ), no Rio de Janeiro-RJ, na época ainda sem respaldo legal. Essa pode ser considerada uma fase experimental da área no Brasil. Uma dificuldade em especial era que o único mercado de trabalho disponível consistia nos próprios serviços que mantinham os PRM. Assim, a maioria dos profissionais formados nestes programas logo considerava a mudança de especialidade, migrando para a saúde pública. ${ }^{5}$

Em 1978, o slogan "Saúde para todos" surgiu durante a Conferência Internacional sobre Atenção Primária à Saúde, organizada pela Organização Mundial da Saúde (OMS) em Alma-Ata. ${ }^{5}$ Os países participantes foram convidados a modificar seus sistemas de saúde de modo a tornar a APS um direito básico dos cidadáos, fato que a expandiu para o mundo. Nesse encontro, discutiu-se o conceito de atenção primária:

"[...] a atenção essencial à saúde baseada num modelo prático, com base científica, que possui métodos e tecnologias universalmente acessíveis aos indivíduos e famílias dentro das comunidades, sendo o primeiro nível de contato do indivíduo com o sistema de saúde do país e condição essencial para o desenvolvimento social e econômico da comunidade (p.27).” ${ }^{\prime 6}$

Apesar do surgimento dos primeiros programas de residência em 1976, somente no ano de 1981 a Comissão Nacional de Residência Médica (CNRM) formaliza os programas de residência médica em Medicina Geral e Comunitária (MGC), oficializando esta como uma especialidade médica no Brasil. Em novembro do mesmo ano, tem-se a criação da Sociedade Brasileira de Medicina Geral e Comunitária (SBMGC). Em 1986, o Conselho Federal de Medicina (CFM) reconhece esta especialidade e a SBMGC como sua representante. ${ }^{5}$

Com a criação do Sistema Único de Saúde (SUS) na Constituição de 1988, posteriormente regulamentado pela Lei 8.080 de 1990 (Lei Orgânica de Saúde), e a implantação do Programa Saúde da Família (PSF) em 1994, a APS se expandiu. ${ }^{6}$ A implantação do PSF procurava reorganizar a atenção em saúde para uma substituição do modelo tradicional, tendo início a construção de um modelo de assistência com base na promoção e proteção da saúde, no diagnóstico precoce e na recuperação dos agravos à saúde, de acordo com as diretrizes e os princípios do SUS.7

O mercado de trabalho para a MGC cresceu, mas existiam poucos especialistas na área e a qualidade da formação era, em muitos casos, abaixo do desejado. Buscando minimizar esses problemas, foram criadas especializaçóes, cursos de curta duração, polos de capacitação, entre outros, nem sempre com a qualidade necessária. ${ }^{6}$ 
Em 2001, ocorreu a mudança do nome da especialidade para Medicina de Família e Comunidade (MFC). No mesmo ano, o Ministério da Saúde implantou o Programa de Interiorização do Trabalho em Saúde (PITS) que propunha Especialização Multiprofissional em Saúde da Família por meio de treinamento em serviço e ensino a distância, coordenado pelos Núcleos e Polos de Capacitação do Programa Agentes Comunitários de Saúde (PACS) e PSF. Esse método foi criticado pelas entidades defensoras da MFC, devido à formação insuficiente de muitos profissionais. ${ }^{5}$

Desde sua criação, o PSF (atualmente Estratégia Saúde da Família) vem se expandindo em todo o País. De acordo com o Ministério da Saúde, existem mais de 34 mil equipes de saúde da família cadastradas no Brasil, no entanto, muitas ainda estão incompletas. Com o aumento de sua dimensão, é crescente a diferença entre a ampliação do mercado de trabalho e a pequena escala de formação e capacitação de profissionais para a APS. ${ }^{8}$

\section{A Atenção Primária à Saúde no contexto atual}

Em 2012, foi implantado o Programa de Valorização da Atenção Básica (PROVAB) e em 2013 o governo federal criou o Programa Mais Médicos, por meio da medida provisória 621 (MP 621). Sáo duas açôes que, apesar de apresentarem grandes dúvidas quanto a seu real impacto na saúde, se mostram marcantes para a APS no País à medida que rompem com a priorização hospitalar e de Unidades de Pronto Atendimento (UPA) que vinha sendo vivenciada. ${ }^{8-10}$

O PROVAB objetiva criar incentivos a médicos, enfermeiros e cirurgióes-dentistas para que atuem na APS de municípios carentes desses profissionais, em áreas de extrema pobreza e periferias das regióes metropolitanas. Ao mesmo tempo, busca consolidar a integração ensino-serviço-comunidade e a educação pelo trabalho, por meio da supervisão dos profissionais por instituiçóes de ensino previamente cadastradas, implantação e manutenção de Núcleos de Telessaúde nas instituições supervisoras e oferecimento de cursos de especializaçáo em Saúde da Família. ${ }^{9}$

Por outro lado, o Programa Mais Médicos tem como finalidade prover recursos humanos na área médica para o Sistema Único de Saúde (SUS) por meio do fornecimento de bolsa e supervisão de instituições de ensino superior para médicos, brasileiros e estrangeiros, que exercerão suas atividades no âmbito de ensino, pesquisa e extensão em locais pré-determinados pelo Ministério da Saúde. Objetiva, ainda, a ampliaçáo de vagas para os cursos de medicina, bem como para programas de residência médica, além de aumentar a inserção do médico em formação nas unidades de atendimento do SUS. ${ }^{10}$

Todas essas são medidas importantes no que diz respeito à valorização da APS, ponto fundamental para se alcançar um sistema de saúde eficaz e de qualidade. Em contrapartida, há uma série de questionamentos que são pertinentes sobre estes programas.

O provimento de médicos para regiôes carentes e distantes dos grandes centros, buscando minimizar as discrepâncias nos serviços de saúde em todo o País, é de fundamental importância, porém é necessário monitorar a qualidade e o perfil desses profissionais, bem como as condiçóes de trabalho oferecidas.

A APS não deve ser vista como algo simples e fácil, trata-se de uma área que requer habilidades e competências especializadas e complexas. Busca-se o atendimento integral à saúde, na qual o personagem central é o individuo, levando-se em consideração o ambiente, os costumes e a realidade na qual está inserido. Faz-se necessário aumentar a capacidade de formação de especialistas em MFC, bem como manter atualizados os profissionais já existentes. Para tanto, seria interessante o aumento das vagas de residência médica nessa área e a criação de meios que incentivassem profissionais a se enveredar por estes caminhos; uma sugestão, por exemplo, seria equiparar as bolsas de residência às bolsas oferecidas aos médicos participantes do PROVAB e Mais Médicos. ${ }^{11,12}$

Outro ponto a ser levado em consideração é a necessidade de políticas que visem à valorização e fixação dos profissionais na APS, não apenas em relação a médicos, mas de toda a equipe multiprofissional. Medidas que melhorariam esse cenário seriam, por exemplo, a abertura de concursos para a contratação desses profissionais e o estabelecimento de pisos salariais, bem como de gratificaçóes, de acordo com o local de trabalho.

A MP 621 não tem como ponto central a fixação dos médicos. Trata-se de uma medida paliativa com prazo para terminar ( 3 anos, podendo se estender por mais 3 anos), o que a torna pouco efetiva. Além disso, não exige formação dos profissionais para a APS, não resolvendo, portanto, o problema de baixa resolutividade e a sobrecarga de alguns serviços em detrimento de outros no SUS. 
O recrutamento de médicos estrangeiros, apesar de ser uma medida adotada em vários países e se mostrar útil e de grande valia, deve ser feito com ponderação e por meio de revalidação do diploma para garantir a segurança da população assistida.

Outro aspecto importante seria aumentar o número de médicos na APS, investindo-se em infraestrutura e insumos, para proporcionar serviços de qualidade. O MFC tem como uma de suas atribuiçôes, por exemplo, fazer alguns procedimentos e pequenas cirurgias, que, muitas vezes, por falta de condiçóes estruturais, se tornam inviáveis e acabam por sobrecarregar outros serviços.

Por fim, a universidade também necessita de uma mudança de visão sobre a APS e a MFC. As escolas médicas não têm conseguido inserir prioritariamente a disciplina da Medicina de Família e Comunidade em seus currículos e sequer proporcionam a quantidade necessária de docentes qualificados para a área. Seria importante, dessa maneira, estimular as instituiçóes de ensino superior para dar respostas adequadas para a resolução destes problemas. ${ }^{13}$

\section{Conclusão}

A MFC, bem como a APS, estiveram sempre à margem dos investimentos em saúde no Brasil em virtude de uma visão hospitalocêntrica e superespecializada. Porém, o País encontra-se em um processo de crescimento e estruturação da MFC, o que representa um terreno fértil para a implementação de ideias e atitudes, com a finalidade de não somente suprir as carências populacionais relacionadas à APS, mas também de criar uma cultura de serviços de qualidade e profissionais preparados para atender a essas demandas.

É de suma importância aproveitar o momento de discussão criado pelos programas do governo (PROVAB e Mais Médicos), que têm como ponto central a APS, para fomentar os incentivos e investimentos nessa área, possibilitando uma reforma no SUS de modo a garantir uma saúde integral e de qualidade à população. Entretanto, uma visão mais ampla a médio e longo prazo é essencial para a sustentabilidade de um projeto de estruturação definitiva da APS no SUS.

\section{Referências}

1. Starfield B. Atenção Primária: equilíbrio entre necessidade de saúde, serviços e tecnologia. Brasília: UNESCO, Ministério da Saúde; 2002.726 p.

2. Janaudis MA. Princípios da Medicina de Família: quatro pilares que definem sua identidade. O Mundo da Saúde. 2010 Jul-Sep;34(3):300-310.

3. Anderson MIP, Gusso G, Castro Filho ED. Medicina de Família e Comunidade: especialistas em integralidade. Revista APS. 2005 Jan-Jun;8(1):61-67.

4. Giovanella L, Mendonça MHM, Almeida PF, Escorel S, Senna MCM, Fausto MCR, et al. Saúde da família: limites e possibilidades para uma abordagem integral de atenção primária à saúde no Brasil. Ciênc Saúde Coletiva. 2009 May-Jun;14(3):783-794. PMid:19547778. http://dx.doi.org/10.1590/ S1413-81232009000300014

5. Falk JW. A Medicina de Família e Comunidade e sua entidade nacional: histórico e perspectivas. Rev Bras Med Fam Comunidade. 2004 Jan-Mar; 1(1):5-10.

6. Janaudis MA, Blasco PG, Haq C, Freeman J. Formando médicos para a Medicina de Família e Comunidade. Revista Bioética. 2007 Jan-Jun;15(1):27-36

7. Ministério da Saúde (BR). Programa de Saúde da Família. Brasília: COSAC; 1994.

8. Ministério da Saúde (BR), SAS, DAB; IBGE. Teto, credenciamento e implantação das estratégias de Agentes Comunitários de Saúde, Saúde da Família e Saúde Bucal. [Internet]. 2013 Sep. [acesso em 2013 Dez 02]. Disponível em: http://dab.saude.gov.br/portaldab/historico_cobertura_sf.php

9. Brasil. Portaria Interministerial 2.087 de 2011. Institui o Programa de Valorização do Profissional da Atenção Básica. [Internet]. [acesso em 2013 Out 9]. Disponível em: http://bvsms.saude.gov.br/bvs/saudelegis/gm/2011/pri2087_01_09_2011.html

10. Brasil. Medida Provisória n 621 de 2013. Institui o Programa Mais Médicos e dá outras providências. [Internet]. [acesso em 2013 Out 10]. Disponível em: http://www.planalto.gov.br/ccivil_03/_ato2011-2014/2013/mpv/mpv621.htm

11. Anderson MIP, Rodrigues RD. Formação de especialistas em Medicina de Família e Comunidade no Brasil: dilemas e perspectivas. Rev Bras Med Fam Comunidade. 2011 Jan-Mar;6(18):19-20. http://dx.doi.org/10.5327/Z1809-59092011001800005

12. Levites M, Blasco PG. Competencia y humanismo: la Medicina Familiar en busca de la excelência [Editorial]. Arch Med Fam General. 2009 Jul-Dez;6(2)2-9. Disponível em: http://archivos.famfyg.org/revista/index.php/amfyg/article/view/88/84

13. Anderson MIP, Demarzo MMP, Rodrigues RD. A Medicina de Família e Comunidade, a Atenção Primária à Saúde e o Ensino de Graduação: recomendações e potencialidades. Rev Bras Med Fam Comunidade. 200 Out-Dez; 3(11):157-172. Disponível em: http://www.rbmfc.org.br/rbmfc/ article/view/334 